\title{
Analysis of Metal Nanoparticles in Biological Tissues Specimens Using Various Surface Analytical Techniques
}

\author{
V.S. Smentkowski, L. Denault, D. Wark \\ General Electric Global Research Center, 1 Research Circle, Niskayuna, NY, 12309 \\ L. Scipioni, D. Ferranti \\ Carl Zeiss SMT, Inc, One Corporation Way, Peabody, MA, 01960
}

Metal based nanoparticles are being evaluated as contrast agents for medical imaging in order to enhance the visibility of internal body structures and processes. Minimally destructive analytical techniques are needed in order to determine where the metal nanoparticles accumulate in treated tissue specimens. The recently introduced Helium Ion Microscope (HIM) offers significant benefits compared to a scanning electron microscope (SEM) for the analysis of biological samples including: (1) higher surface specificity, (2) reduction of surface charging effects during analysis of insulating samples (for many biological samples placed onto a glass substrate, a conductive overlayer is not needed), (3) higher depth of field, and (4) better lateral resolution. Unfortunately, for many material systems, the HIM is not (yet) able to provide chemical analysis.

We have recently analyzed spleen tissue specimens from a rat that was injected with metal based nanoparticles and from a control animal that was not injected with metal nanoparticles. The samples were prepared by fixing in $10 \%$ formalin for approximately 24 hours then dehydrating in a series of ethanol baths to finally embed in paraffin wax. Tissues were sliced 5 microns thick and collected onto standard, positively charged microscope slides. Some of the slides were rinsed in xylene to remove some of the paraffin wax from the tissue.

FIG 1 shows HIM images; treated is on the left and the untreated/control is on the right. The image on the left clearly shows contrast that is not observed in the image on the right (untreated). HIM analysis of treated samples that were not rinsed with xylene (not shown here) did not reveal contrast, indicating that the xylene rinse removed surface contaminants that originated from the embedding in paraffin wax; demonstrating that xylene rinsing of biological samples may be a critical step needed for HIM analysis of paraffin wax imbedded tissue samples. As was mentioned above, HIM analysis is not able to provide direct chemical analysis, hence a second analysis technique was desired that exhibits high surface specificity, chemical sensitity, and is able to provide high lateral resolution imaging without coating of the samples. Time of flight secondary ion mass spectrometry (ToF-SIMS) is a technique that meets these requirements. Typically, the high current bunhced mode is used to perform both high mass resolution and the highest sensitivity ToF-SIMS analysis, at the expense of lateral resolution. In order to improve the lateral resolution, the buncher is often turned off which reduces the mass resolution; unfortunately this prevents the positive identification of metals in tissue samples due to the overlap of biological fragments (CxHy) and the metal signal which are both at the same nominal mass. The ToF-SIMS burst mode of analysis enables high lateral resolution and high mass resolution ToF-SIMS analysis, but results in low data collection rates which means that the images need to be signal averaged longer; the burst mode of analysis was utilized in this work. FIG 2 shows burst mode ToF-SIMS analysis of the metal contrast agent employed, the distribution of metal is similar to that measured using HIM, however the ToF-SIMS image reveals lower signal and overall contrast since the sampling depth of 
ToF-SIMS is less than that of HIM. ToF-SIMS analysis of the untrated sample revealed significantly less metal.

In the next few weeks, these samples will be coated, and analyzed by more conventional techniques including SEM/EDS and electron microprobe (WDS) in order to provide for a thorough understanding of the distribution of the metal contrast agent in the sample. This presentation demonstrates the benefits of HIM for the analysis of treated tissue samples, and shows that the data generated from complimentary characterization tools provides for a more thorough understanding and complete description of the sample which is superior to the data collected using only one characterization technique.
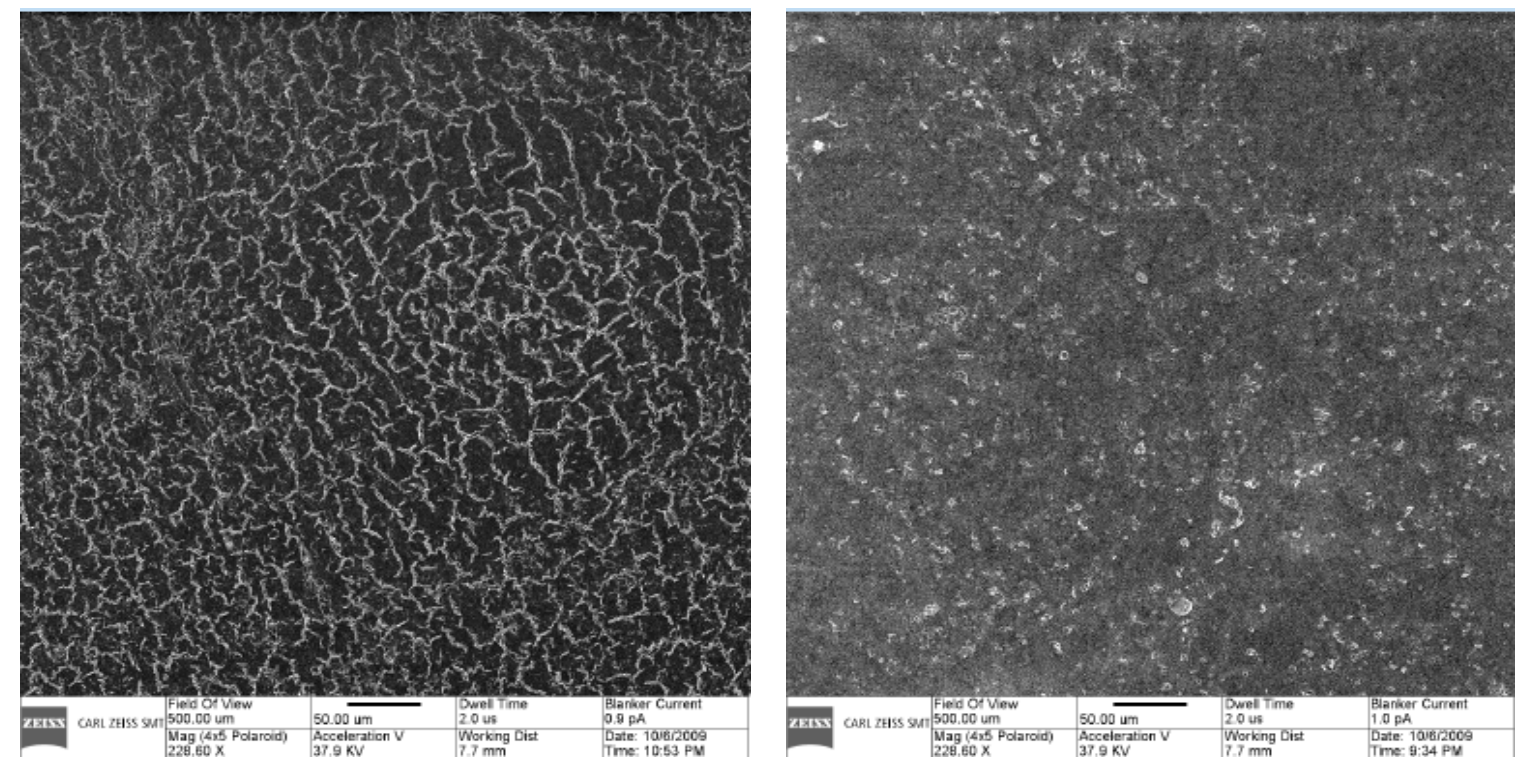

FIG 1. HIM images of treated (left) and untreated (right) spleen tissue samples. The treated sample shows contrast that is not observed in the untreated sample and is attributed to the metal contrast agent.
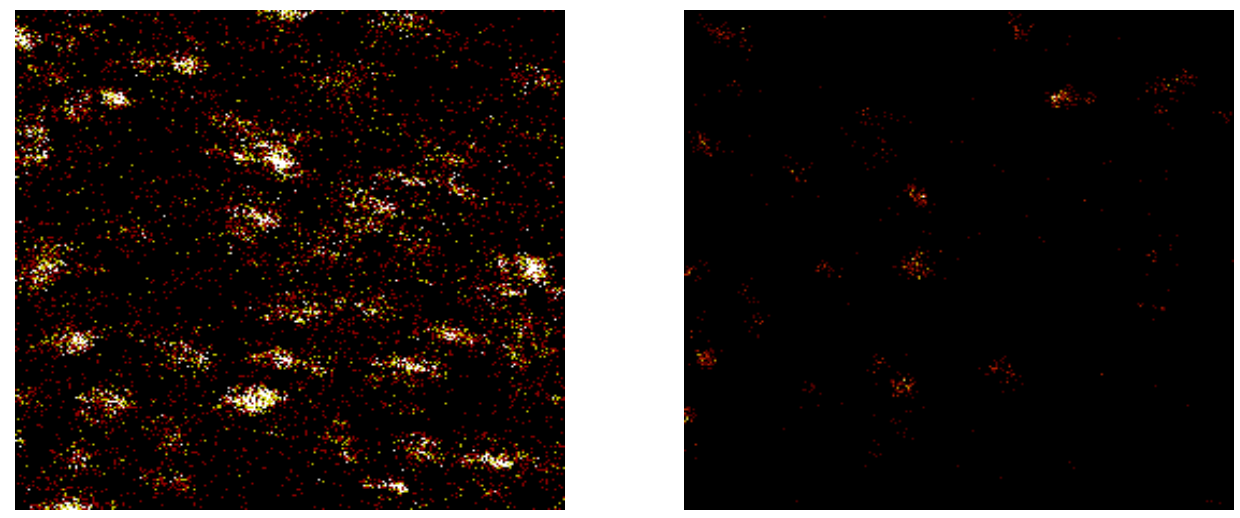

FIG 2. ToF-SIMS elemental images of the metal contrast agent used in the treated (left) and untreated (right) spleen tissue samples. ToF-SIMS provides chemical information that is lacking in the HIM images. Area analyzed by ToF-SIMS is 146 microns by 146 microns. 